\title{
Bevacizumab-containing regimens after cetuximab failure in Kras wild-type metastatic colorectal carcinoma
}

\author{
KA ON LAM ${ }^{1,2}$, VICTOR HO FUN LEE ${ }^{1,2}$, RICO KIN YIN LIU ${ }^{2}$, TO WAI LEUNG ${ }^{2}$ and DORA LAI WAN KWONG ${ }^{1,2}$ \\ ${ }^{1}$ Department of Clinical Oncology, Faculty of Medicine, The University of Hong Kong; \\ ${ }^{2}$ Department of Clinical Oncology, Queen Mary Hospital, Hong Kong, SAR, P.R. China
}

Received August 13, 2012; Accepted November 13, 2012

DOI: 10.3892/ol.2012.1045

\begin{abstract}
Bevacizumab and cetuximab both improve treatment efficacy when administered with chemotherapy for metastatic colorectal carcinoma (mCRC). Cetuximab has enhanced efficacy in Kras wild-type tumors. However, inferior outcomes have been demonstrated concerning the concurrent use of bevacizumab and cetuximab with chemotherapy. There is an urgent need to define the optimal sequence of use of these two agents. With regard to the pre-clinical data that increased VEGF expression is associated with acquired resistance to anti-EGFR antibody, we performed a retrospective analysis on the outcomes of patients who received bevacizumab-containing regimens after cetuximab failure in Kras wild-type mCRC. From January 2006 to December 2011, patients who received bevacizumab-containing regimens for $\mathrm{mCRC}$ in our institution were reviewed. Patients were eligible for further analysis if the following criteria were met: i) Kras wild-type mCRC; ii) chemotherapy and cetuximab received as immediate prior treatment; iii) chemotherapy and bevacizumab received as the index line of treatment; and iv) imaging conducted for response evaluation. Outcome measures included median progression-free survival (mPFS) and objective response rate (ORR). Targeted adverse events were recorded in accordance with two prospective observational cohort studies; the BRiTE and BEAT studies. Fifty patients who received bevacizumab-containing regimens were reviewed and 18 of them met the criteria for further analysis. After a median follow-up of 12.1 months, the mPFS for the total group of patients was 26.3 weeks (95\% CI, 19.5-33.0 weeks) with an ORR of $38.9 \%$. Two patients $(11.1 \%)$ had hypertension that required additional anti-hypertensive drugs and one patient did not survive due to a bowel perforation. No arterial throm-
\end{abstract}

Correspondence to: Dr Ka On Lam, 1/F Professorial Block, Department of Clinical Oncology, Queen Mary Hospital, Pokfulam Road, Hong Kong, SAR, P.R. China

E-mail: lamkaon@hku.hk

Key words: bevacizumab, cetuximab, metastatic colorectal carcinoma, Kras wild-type boembolic events (ATEs), post-operative wound-healing complications (POWHCs) or grade III/IV bleeding were observed. In patients with Kras wild-type mCRC, bevacizumab-containing regimens following cetuximab failure have modest activity and manageable toxicity.

\section{Introduction}

Colorectal carcinoma is one of the most common malignancies worldwide. Prior to the era of targeted therapy, chemotherapy was the only option of systemic therapy for metastatic colorectal carcinoma (mCRC). Chemotherapy regimens, comprising 5-fluorouracil (5-FU) plus leucovorin (LV) backbone with either oxaliplatin or irinotecan, have improved both progression-free survival (PFS) and overall survival (OS) (1-4). Capecitabine, an oral antimetabolite that is metabolised preferentially in tumor cells, in combination with oxaliplatin demonstrates a similar efficacy to FOLFOX4 (oxaliplatin in combination with 5-FU and LV) in the treatment of mCRC (5).

The development of targeted therapy has expanded treatment options for patients with mCRC. Bevacizumab, a recombinant humanized monoclonal antibody against vascular endothelial growth factor (VEGF), used in conjunction with chemotherapy has demonstrated efficacy as a first- and secondline treatment (6-8). On the other hand, cetuximab, a chimeric monoclonal antibody against epithelial growth factor receptor (EGFR), is active as a first- and second-line treatment when combined with chemotherapy and as a single agent in thirdline therapy (9-11). It has enhanced the effect of treatment in patients with Kras wild-type mCRC $(9,12)$ and testing for the Kras status of the tumor specimen is recommended in various international treatment guidelines.

With regard to chemotherapy, the efficacy is independent of the sequence of use of individual chemotherapeutic agents, provided that patients are treated with all the active agents $(13,14)$. However, the same principle of maximum exposure and indiscriminate sequence of use of all agents may not be applicable to the use of anti-VEGF and anti-EGFR antibodies (15-17). In the case of cetuximab failure, the option of either switching to a bevacizumab-containing regimen or using cetuximab beyond progression are both practiced but lack supporting evidence. As pre-clinical data have suggested that acquired resistance to anti-EGFR antibody is associated 
with an increased level of VEGF, the sequence of their use may have practical implications (18-20). In this retrospective study, the outcomes of patients who received bevacizumabcontaining regimens following cetuximab failure for Kras wild-type mCRC were reviewed and presented.

\section{Materials and methods}

Study eligibility. All patients with $\mathrm{mCRC}$ who were treated with bevacizumab-containing regimens between January 2006 and December 2011 were screened. Patients were eligible for review in our study if they met the following criteria: i) Kras wild-type mCRC; ii) chemotherapy and cetuximab received as immediate prior treatment; iii) chemotherapy and bevacizumab received as the index line of treatment; and iv) imaging conducted for response evaluation. Out of the 50 patients that were screened, 18 patients satisfied the criteria and were eligible for analysis.

Chemotherapy regimens. Patients were treated with bevacizumab at $5 \mathrm{mg} / \mathrm{kg}$ every 2 weeks if combined with FOLFOX4 (1) or FOLFIRI (irinotecan plus 5-FU and LV) (2), or at $7.5 \mathrm{mg} /$ $\mathrm{kg}$ every 3 weeks if combined with XELOX (capecitabine plus oxaliplatin) (5), XELIRI (capecitabine plus irinotecan) (21) or XELODA (capecitabine alone) (22). No dose adjustment was permitted for bevacizumab, while the dose of chemotherapeutic agents was determined and adjusted at the discretion of the treating oncologist, based on our departmental protocol.

Outcomes measures. Outcome measures included PFS (from the start of bevacizumab treatment following cetuximab failure, to the first recorded occurrence of physician-assessed disease progression, $\mathrm{PD}$, or death). The objective response rate (ORR) was evaluated by imaging using response evaluation criteria in solid tumors (RECIST) criteria every 8-12 weeks of treatment (23).

Targeted adverse events were recorded in accordance with two prospective observational cohort studies, the BRiTE and BEAT study $(24,25)$. Adverse events included gastrointestinal perforation (GIP; perforation, intra-abdominal abscess and fistula), arterial thromboembolic events (ATEs; myocardial infarction, cerebrovascular accident, transient ischemic attack and unstable angina), postoperative bleeding or woundhealing complications (POWHCs), grade III/IV bleeding and hypertension requiring additional anti-hypertensives. Toxicity grading was based on the National Cancer Institute (NCI) Common Toxicity Criteria for Adverse Events (CTCAE), version 3.0 (26). Adverse events attributed to bevacizumab were identified up to 90 days after permanent discontinuation of the drug.

Statistical analysis. The primary endpoint of our analysis was median progression-free survival (mPFS) and ORR. Survival rates were estimated using the Kaplan-Meier method and survival curves were compared using the log-rank test. The Fisher's exact test was used to compare response rates. $\mathrm{P}<0.05$ was considered to indicate a statistically significant difference. Analyses were conducted using the Statistical Package for Social Sciences (SPSS) 19.0 for Windows (SPSS, Inc.; Chicago, IL, USA).
Table I. Baseline patient characteristics.

\begin{tabular}{|c|c|}
\hline Characteristic & $\begin{array}{l}\text { No. patients } \\
\qquad(\mathrm{n}=18)\end{array}$ \\
\hline Median age (range) & $56.5(42-72)$ \\
\hline \multicolumn{2}{|l|}{ Gender } \\
\hline Male & 9 \\
\hline Female & 9 \\
\hline \multicolumn{2}{|c|}{ ECOG performance status } \\
\hline 0 & 3 \\
\hline 1 & 15 \\
\hline \multicolumn{2}{|l|}{ Primary tumor site } \\
\hline Colon & 13 \\
\hline Rectum & 5 \\
\hline \multicolumn{2}{|c|}{ Number of metastatic sites } \\
\hline 1 & 8 \\
\hline$>1$ & 10 \\
\hline \multicolumn{2}{|l|}{ Site of metastasis } \\
\hline Liver & 15 \\
\hline Lymph node & 8 \\
\hline Lung & 5 \\
\hline Locoregional & 4 \\
\hline Peritoneum & 3 \\
\hline \multicolumn{2}{|l|}{ Prior chemotherapy } \\
\hline Fluoropyrimidine & 18 \\
\hline Oxaliplatin & 17 \\
\hline Irinotecan & 6 \\
\hline
\end{tabular}

\section{Results}

Patient characteristics. The median patient age was 56.5 years. Metastatic disease was identified at initial diagnosis in 15 $(83.3 \%)$ patients, while $10(55.6 \%)$ patients presented with metastases involving more than 1 organ. The liver was the most common site of metastasis and 5 patients exhibited liver-only metastasis (Table I). Following cetuximab failure, 8 and 10 patients received second- and third-line bevacizumab-containing regimens, respectively. Bevacizumab was administered with irinotecan-based chemotherapy in 13 patients and oxaliplatin-based chemotherapy in 5 patients. The median time period from cetuximab failure to the start of bevacizumab treatment was 6.8 weeks (range, 1-60) and the median number of cycles of bevacizumab was 6.5 (range, 4-12).

Treatment efficacy. After a median follow-up of 12.1 months, the mPFS for the total group of patients was 26.3 weeks (95\% CI, 19.5-33.0) with an ORR of 38.9\%. For the 8 patients who received bevacizumab-containing regimens as a secondline treatment, 1 complete response (CR) and 3 partial responses (PR) were observed, producing an ORR of $50 \%$. The mPFS was 27.4 weeks $(95 \%$ CI, 2.0-52.8). For the 10 patients who received the third-line treatment, 3 PRs were observed and thus the ORR was $30 \%$. The mPFS was 23.9 weeks 


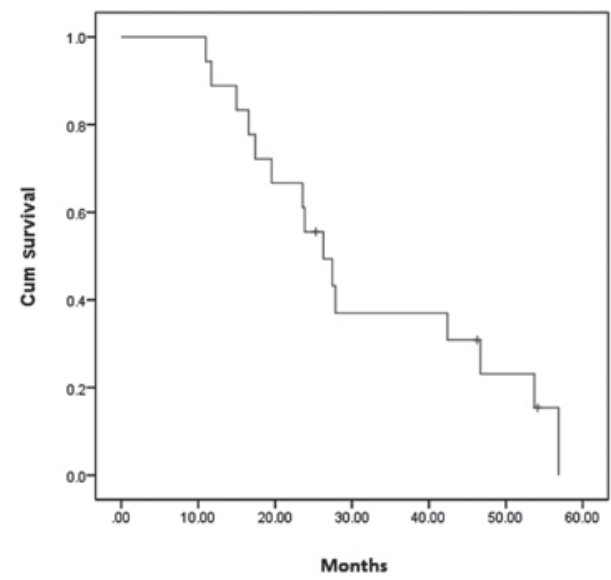

Figure 1. Kaplan-Meier plot of progression-free survival for all patients receiving bevacizumab-containing regimens following cetuximab failure.

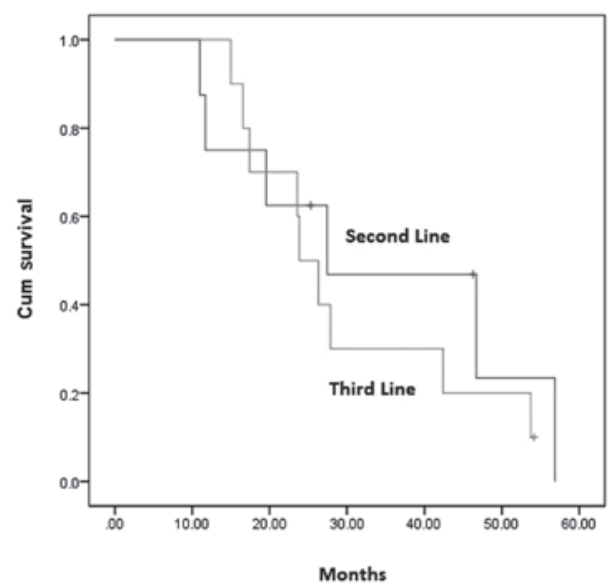

Figure 2. Kaplan-Meier plot of progression-free survival and patients receiving bevacizumab-containing regimens as second- and third-line therapy.

(95\% CI, 19.7-28.1). No statistically significant difference in PFS $(\mathrm{P}=0.552)$ and ORR $(\mathrm{P}=0.63)$ was observed between patients who received bevacizumab as a second- or third-line treatment following cetuximab failure (Figs. 1 and 2).

Toxicity related to bevacizumab. Two patients presented with worsened hypertension that was controlled by an additional anti-hypertensive drug. One patient was found to have intestinal perforation 78 days after the last dose of bevacizumab. Another patient had bevacizumab suspended for 6 weeks before planned resection of liver and pelvic metastases. Complete resection was achieved and pathological examination confirmed a partial response. Additionally, no post-operative complications were observed for this patient. In the whole cohort of patients, no ATEs or grade III/IV bleeding were observed.

\section{Discussion}

To our knowledge, this is the first study concerning the outcomes of bevacizumab-containing regimens following cetuximab failure in patients with Kras wild-type mCRC.
The treatment outcomes of patients treated with bevacizumab-containing regimens as the second-line therapy were comparable with a previously reported phase III trial (8). Patients treated with bevacizumab-containing regimens as the third-line therapy demonstrated a median PFS of 23.9 weeks and an ORR of $30 \%$, which were both superior to those found previously. Emmanouilides et al studied the outcomes of 19 patients who had received bevacizumab with 5-FU plus LV as a third-line treatment in a prospective study. The median time to progression was 16 weeks but no objective response was documented (27). In a retrospective analysis by Kang et al, bevacizumab was combined with either FOLFOX or FOLFIRI in a third-line or later treatment after failure of 5-FU, oxaliplatin and irinotecan. The median PFS was 5.3 months and the overall response rate was 9.5\% (28). The results of these two studies were similar to those of a TRC-0301 study in which the median PFS was 3.5 months and the response rate was $4 \%$ (29). Vincenzi et al conducted a phase II study using bevacizumab and 5-FU plus LV as the fourth-line setting in 48 patients who failed cetuximab, oxaliplatin, irinotecan and 5-FU treatment. The response rate was only $6.25 \%$; however, $30.4 \%$ of patients achieved a stable disease status (30). In all the aforementioned studies, the Kras status of tumors was not noted and prior cetuximab exposure was only documented in the study by Vincenzi et al. Although 17 patients in the present study failed oxaliplatin, 5-FU and cetuximab treatment, only 6 patients in our study as compared with all patients in the aforementioned studies failed oxaliplatin, irinotecan and 5-FU treatment. While 'chemo-refractoriness' differed among patients in the present study and those quoted previously, the potential additional benefit of using bevacizumab following cetuximab failure should not be overlooked.

It has been demonstrated that the exact sequence of chemotherapeutic agents used in mCRC chemotherapy did not affect the outcome (14), provided patients were exposed to all active agents (13). However, there is no real evidence for applying the same principle to the use of anti-EGFR and anti-VEGF antibodies. Both the CAIRO2 and PACCE trials demonstrated inferior results with the addition of anti-EGFR antibody to bevacizumab-containing regimens $(15,16)$. These two phase III randomized controlled studies were unable to confirm why administering more did not lead to improvement with regard to the use of targeted therapies; however, the results did call for the investigation of an optimal sequence of use of these targeted therapies. Notably, a study by Norguet et al investigated the effect of prior exposure to bevacizumab on the efficacy of subsequent cetuximab treatment (17). In the present study, patients with prior exposure to bevacizumab were associated with a significantly inferior outcome with subsequent cetuximab treatment. Taken together, it is necessary to identify patients who may benefit most from a specific sequence of use of anti-EGFR and anti-VEGF antibodies.

An enhanced treatment effect was demonstrated in patients with Kras wild-type tumor treated with cetuximab $(9,12)$. All patients in the present study had Kras wild-type tumor and were treated with cetuximab. As demonstrated in pre-clinical studies, prolonged exposure of cancer cells to EGFR-blocking antibodies gives rise to resistant cells that have increased VEGF expression. Thus, cancer cells may become more 
dependent on the VEGF pathway when they acquire resistance to the EGFR inhibitor (18-20). It could be postulated that the superior outcomes of the patients in the present study were partly due to the selection of patients with Kras wild-type tumor; patients were treated with bevacizumab at a time when the cancer cells had become more dependent on the VEGF pathway upon acquiring resistance to the EGFR inhibitor.

In the present study, the toxicity related to bevacizumab was infrequent and manageable. Two patients required administration of one additional anti-hypertensive drug for the treatment of worsened hypertension during the course of bevacizumab. This proportion of patients was similar to those observed in landmark studies with an incidence of $4-11 \%$ for grade III/IV hypertension (6-8). The patient who did not survive due to a bowel perforation was unlikely to have suffered the perforation as a result of bevacizumab; the event occurred 78 days after the last dose of bevacizumab when the patient was receiving hypofractionated palliative radiotherapy to the pelvis. Therefore, there were no patient fatalities due to bevacizumab-related toxicity during the active phase of bevacizumab treatment.

In conclusion, the use of bevacizumab-containing regimens following cetuximab failure in patients with Kras wild-type mCRC has modest activity and acceptable toxicity. A small sample size and retrospective nature were the major limitations of the present study. However, the results remain informative. Unlike concurrent use of bevacizumab and cetuximab, and likely the sequential use of cetuximab following bevacizumab, the use of bevacizumab-containing regimens following cetuximab failure may represent an optimal sequence of targeted therapies and warrants further research in prospective studies.

\section{References}

1. de Gramont A, Figer A, Seymour M, et al: Leucovorin and fluorouracil with or without oxaliplatin as first-line treatment in advanced colorectal cancer. J Clin Oncol 18: 2938-2947, 2000.

2. Douillard JY, Cunningham D, Roth AD, et al: Irinotecan combined with fluorouracil compared with fluorouracil alone as first-line treatment for metastatic colorectal cancer: a multicentre randomised trial. Lancet 355: 1041-1047, 2000.

3. Goldberg RM, Sargent DJ, Morton RF, et al: A randomized controlled trial of fluorouracil plus leucovorin, irinotecan, and oxaliplatin combinations in patients with previously untreated metastatic colorectal cancer. J Clin Oncol 22: 23-30, 2004.

4. Saltz LB, Cox JV, Blanke C, et al: Irinotecan plus fluorouracil and leucovorin for metastatic colorectal cancer. N Engl J Med 343: 905-914, 2000

5. Cassidy J, Clarke S, Díaz-Rubio E, et al: Randomized phase III study of capecitabine plus oxaliplatin compared with fluorouracil/folinic acid plus oxaliplatin as first-line therapy for metastatic colorectal cancer. J Clin Oncol 26: 2006-2012, 2008 .

6. Hurwitz H, Fehrenbacher L, Novotny W, et al: Bevacizumab plus irinotecan, fluorouracil, and leucovorin for metastatic colorectal cancer. N Engl J Med 350: 2335-2342, 2004.

7. Saltz LB, Clarke S, Díaz-Rubio E, et al: Bevacizumab in combination with oxaliplatin-based chemotherapy as first-line therapy in metastatic colorectal cancer: a randomized phase III study. J Clin Oncol 26: 2013-2019, 2008.

8. Giantonio BJ, Catalano PJ, Meropol NJ, et al: Bevacizumab in combination with oxaliplatin, fluorouracil, and leucovorin (FOLFOX4) for previously treated metastatic colorectal cancer: results from the Eastern Cooperative Oncology Group Study E3200. J Clin Oncol 25: 1539-1544, 2007.

9. Van Cutsem E, Köhne CH, Hitre E, et al: Cetuximab and chemotherapy as initial treatment for metastatic colorectal cancer. N Engl J Med 360: 1408-1417, 2009.
10. Cunningham D, Humblet $Y$, Siena S, et al: Cetuximab monotherapy and cetuximab plus irinotecan in irinotecan-refractory metastatic colorectal cancer. N Engl J Med 351: 337-345, 2004.

11. Jonker DJ, O'Callaghan CJ, Karapetis CS, et al: Cetuximab for the treatment of colorectal cancer. N Engl J Med 357: 2040-2048, 2007.

12. Bokemeyer C, Bondarenko I, Hartmann JT, et al: Efficacy according to biomarker status of cetuximab plus FOLFOX-4 as first-line treatment for metastatic colorectal cancer: the OPUS study. Annals of Oncology 22: 1535-1546, 2011.

13. Grothey A, Sargent D, Goldberg RM and Schmoll HJ: Survival of patients with advanced colorectal cancer improves with the availability of fluorouracil-leucovorin, irinotecan, and oxaliplatin in the course of treatment. J Clin Oncol 22: 1209-1214, 2004.

14. Tournigand C, André T, Achille E, et al: FOLFIRI followed by FOLFOX6 or the reverse sequence in advanced colorectal cancer: a randomized GERCOR study. J Clin Oncol 22: 229-237, 2004.

15. Tol J, Koopman M, Cats A, et al: Chemotherapy, bevacizumab, and cetuximab in metastatic colorectal cancer. N Engl J Med 360: 563-572, 2009.

16. Hecht JR, Mitchell E, Chidiac T, et al: A randomized phase IIIB trial of chemotherapy, bevacizumab, and panitumumab compared with chemotherapy and bevacizumab alone for metastatic colorectal cancer. J Clin Oncol 27: 672-680, 2009.

17. Norguet E, Dahan L, Gaudart J, Gasmi M, Ouafik Lh and Seitz JF: Cetuximab after bevacizumab in metastatic colorectal cancer: Is it the best sequence? Dig Liver Dis 43: 917-919, 2011.

18. Bianco R, Rosa R, Damiano V, et al: Vascular endothelial growth factor receptor-1 contributes to resistance to anti-epidermal growth factor receptor drugs in human cancer cells. Clin Cancer Res 14: 5069-5080, 2008.

19. Naumov GN, Nilsson MB, Cascone T, et al: Combined vascular endothelial growth factor receptor and epidermal growth factor receptor (EGFR) blockade inhibits tumor growth in xenograft models of EGFR inhibitor resistance. Clin Cancer Res 15: 3484-3494, 2009.

20. Viloria-Petit A, Crombet T, Jothy S, et al: Acquired resistance to the antitumor effect of epidermal growth factor receptor-blocking antibodies in vivo. Cancer Res 61: 5090-5101, 2001.

21. Patt YZ, Lee FC, Liebmann JE, et al: Capecitabine plus 3-weekly irinotecan (XELIRI regimen) as first-line chemotherapy for metastatic colorectal cancer: phase II trial results. Am J Clin Oncol 30: 350-357, 2007.

22. Cassidy J, Twelves C, Van Cutsem E, et al: First-line oral capecitabine therapy in metastatic colorectal cancer: a favorable safety profile compared with intravenous 5-fluorouracil/leucovorin. Annals of Oncology 13: 566-575, 2002.

23. Eisenhauer EA, Therasse P, Bogaerts J, et al: New response evaluation criteria in solid tumours: Revised RECIST guideline (version 1.1). Eur J Cancer 45: 228-247, 2009.

24. Kozloff M, Yood MU, Berlin J, et al: Clinical outcomes associated with bevacizumab-containing treatment of metastatic colorectal cancer: the BRiTE observational cohort study. Oncologist 14: 862-870, 2009.

25. Van Cutsem E, Rivera F, Berry S, et al: Safety and efficacy of first-line bevacizumab with FOLFOX, XELOX, FOLFIRI and fluoropyrimidines in metastatic colorectal cancer: the BEAT study. Ann Oncol 20: 1842-1847, 2009.

26. Trotti A, Colevas AD, Setser A, et al: CTCAE v3.0: development of a comprehensive grading system for the adverse effects of cancer treatment. Semin Radiat Oncol 13: 176-181, 2003.

27. Emmanouilides C, Pegram M, Robinson R, Hecht R, Kabbinavar F and Isacoff W: Anti-VEGF antibody bevacizumab (Avastin) with 5FU/LV as third line treatment for colorectal cancer. Tech Coloproctol 8: s50-52, 2004.

28. Kang B, Kim T, Lee JL, et al: Bevacizumab plus FOLFIRI or FOLFOX as third-line or later treatment in patients with metastatic colorectal cancer after failure of 5-fluorouracil, irinotecan, and oxaliplatin: a retrospective analysis. Med Oncol 26: 32-37, 2009.

29. Chen HX, Mooney M, Boron M, et al: Phase II multicenter trial of bevacizumab plus fluorouracil and leucovorin in patients with advanced refractory colorectal caner: an NCI Treatment Referral Center Trial TRC-0301. J Clin Oncol 24: 3354-3360, 2006.

30. Vincenzi B, Santini D, Russo A, et al: Bevacizumab in association with de Gramont 5-fluorouracil/folinic acid in patients with oxaliplatin-, irinotecan-, and cetuximab-refractory colorectal cancer: a single-center phase 2 trial. Cancer 115: 4849-4856, 2009. 